\title{
Short distance physics with heavy quark potentials *
}

\author{
F. Zantow with O. Kaczmarek, F. Karsch, P. Petreczky \\ Fakultät für Physik, Universität Bielefeld, \\ D-33615 Bielefeld, Germany
}

\begin{abstract}
We present lattice studies of heavy quark potentials in the quenched approximation of QCD at finite temperatures. Both, the color singlet and color averaged potentials are calculated. While the potentials are well known at large distances, we give a detailed analysis of their short distance behavior (from $0.015 \mathrm{fm}$ to $1 \mathrm{fm}$ ) near the critical temperature. At these distances we expect that the T-dependent potentials go over into the zero temperature potential. Indeed, we find evidences that the temperature influence gets suppressed and the potentials starts to become a unique function of the underlying distance scale. We use this feature to normalize the heavy quark potentials at short distances and extract the free energy of the quark system in a gluonic heat bath.
\end{abstract}

\section{INTRODUCTION}

The study of heavy quark potentials (free energies) is important for our understanding of the fundamental forces in strongly interacting matter. For example it finds applications in the phenomenology of heavy quarkonium physics at finite temperature $[1,2,3]$. Our aim is to get deeper insights in their short distance behavior $(R<0.1 \mathrm{fm})$. It is expected that at distances $R<<1 / T$ the presence of a thermal medium does not modify physics. However the question arises at which distance scales the temperature effects become negligible.

We performed lattice studies of the color averaged and the color singlet heavy quark free energy. Our calculations have been performed on a $32^{3} \times 8$ and $32^{3} \times 16$ lattice using a tree-level Symanzik improved action and on a $64^{3} \times 16$ lattice using the standard Wilson action of the pure $S U(3)$ theory. Up to an additive renormalization, the color averaged free energy, $F_{a v}:=F_{a v}(R, T)$, is defined in terms of Polyakov loop correlation functions [4] $F_{a v}=-T \ln \left\langle\tilde{\operatorname{Tr}} L(\overrightarrow{0}) \tilde{\operatorname{Tr}} L^{\dagger}(\vec{R})\right\rangle+C_{a v}$, where $L$ denotes the Polyakov loop and $\tilde{T}$ is the normalized trace: $\tilde{\operatorname{Tr}} \equiv 1 / N_{c}$ Tr. Following Ref. [5] the color singlet free energy $F_{s}:=$ $F_{s}(R, T)$ is identified with the cyclic Wilson loop

${ }^{*}$ This work was supported by DFG Grant No. FOR 339/12 . via $F_{s}=-T \ln \left\langle\tilde{\operatorname{Tr}} L(\overrightarrow{0}) D_{\vec{R}} L^{\dagger}(\vec{R}) D_{\vec{R}}^{\dagger}\right\rangle+C_{s}$, where $D_{\vec{R}}$ is the product of links on the shortest connection from $\overrightarrow{0}$ to $\vec{R}$. This formula is expected to be valid at short distances [5]. The renormalization constants $C_{a v}$ and $C_{s}$ contain temperature independent divergent self-energy contributions. As no additional divergences are introduced at $T>0$ we can fix the renormalization constants, if we are able to separate the $T$-independent part in the free energy and match it to the zero temperature heavy quark potential.

The color averaged free energy can be related to the singlet $\left(F_{s}\right)$ and octet $\left(F_{o}\right)$ contributions due to:

$$
\begin{aligned}
& \exp \left(-F_{a v} / T\right) \\
& =1 / 9 \exp \left(-F_{s} / T\right)+8 / 9 \exp \left(-F_{o} / T\right) .
\end{aligned}
$$

In a static system the internal energy reduces to the potential $V$ and thus the free energy refers to $F=V-T S$, where $S$ denotes the entropy. In a perturbative treatment of QCD at lowest order the singlet and octet potentials are of Coulomb form [6]:

$V_{s}(R)=-\frac{4}{3} \frac{g^{2}}{4 \pi R}, \quad V_{o}(R)=-\frac{1}{8} V_{s}(R)$,

where $g$ is the QCD running coupling. A high temperature expansion of (1) in combination with (2) yields the color averaged heavy quark poten- 
tial:

$\frac{V(R, T)}{T}=-\frac{1}{9} \frac{g^{4}}{(4 \pi)^{2}} \frac{1}{(R T)^{2}}=-\frac{\alpha_{s}^{2}}{9(R T)^{2}}$,

with $\alpha_{s}=g^{2} /(4 \pi)$. Note that (3) is restricted to large distances in order to justify the high temperature expansion. At short distances the octet contribution in (1) is suppressed leading to a singletlike behavior of the color averaged potential [7].

\section{THE COLOR AVERAGED POTEN- TIAL AT $T>T_{c}$}

We study the short distance behavior of the color averaged potential in terms of the screening function $S(R, T)$ defined by $S(R, T)=$ $-9 V(R, T) T[1 / R]^{-2}$, with $\left[\frac{1}{R}\right]$ being the lattice Coulomb potential. At large distances the screening function is exponentially suppressed (hence its name) and in the regime where the perturbative expression (3) is applicable we expect $S=\alpha_{s}^{2}$. In Fig. 1 the square root of the screening function is plotted. Two distinct features are evident from our data: First, the screening function is decreasing at very short distances $(R T \lesssim 0.1)$ indicating the singlet-like behavior mentioned before. Secondly, screening effects become important at rather short distances (about 0.3/T). Nonetheless, there still remain temperature effects in the color averaged potentials at short distances.

We have also compared the lattice data with a 1-loop perturbative calculation (up to $\mathcal{O}\left(g^{6}\right)$ ) in Fig. 1. In this calculation we have added the non-static contributions to the potential which are expected to become important in the short distance regime (see Appendix A). As one can see from Fig. 1 the color averaged potential can be described by 1-loop perturbation theory for $T \gtrsim 6 T_{c}$ and distances $0.3 \lesssim R T \lesssim 1$. For smaller temperatures and larger distances perturbation theory breaks down due to non-perturbative effects. Our calculation also breaks down at very small distances $R T \lesssim 0.1$ because of the enclosed high temperature expansion.

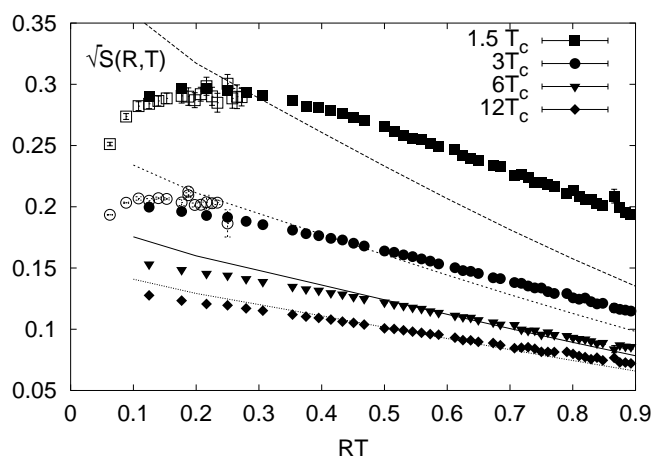

Figure 1. The square root of the screening function $\sqrt{S}$ calculated on a $32^{3} \times 8$ lattice (filled symbols) and on a $32^{3} \times 16$ (or $64^{3} \times 16$ ) lattice (open symbols) and the corresponding perturbative results (lines).

\section{THE TEMPERATURE INDEPEN- DENCE AT SHORT DISTANCES}

In order to eliminate effects from color averaging we now refer to the short distance behavior of the color singlet potentials. Our results together with the zero temperature potential from [8] are shown in Fig 2. The potentials have been normalized at short distances to make a comparison with the zero temperature limit possible. As one can see from Fig. 2 the temperature dependence of the heavy quark potential becomes first visible at distances of about $R \gtrsim 0.1 \mathrm{fm}$. Thus the (finite $T$ ) color singlet potentials approach their zero temperature limit already at the plotted distances. Indeed this analysis provides evidence that the running coupling extracted from color singlet data using (2) starts to get $T$-independent at these distances.

We now argue that these normalized data refer to the free energy of the estimated quark system enclosing effects from the gluonic heat bath - because divergent self-energy contributions cancel out. Moreover we expect that the free energy has a well defined continuum limit in our approach because the normalization gets independent of the lattice cut-off at sufficiently short distances (see Fig. 2). 


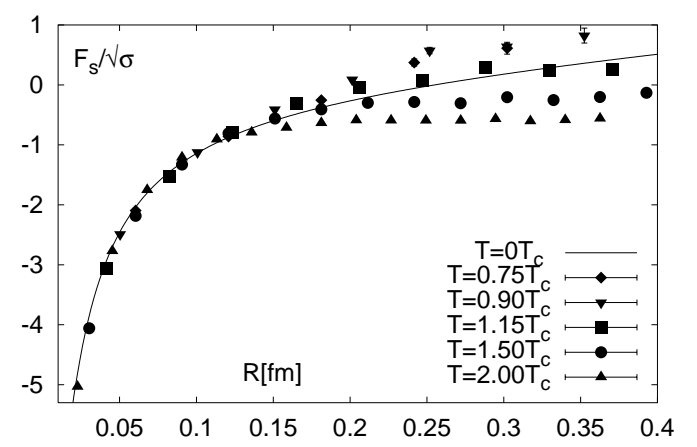

Figure 2. The zero temperature limit of color singlet potentials at non-zero temperature.

\section{CONCLUSIONS}

Our study shows that the heavy quark potentials have a non-trivial structure at short distances. Color averaging influences the physics of potentials at short distances and causes that the averaged data approach the $T=0$ limit at shorter distances as do the singlet data. The color singlet potentials approach the zero temperature limit at distances $R \sim 0.1 \mathrm{fm}$ and $T \sim T_{c}$. Due to this feature we have extracted the free energy of a heavy quark pair enclosing the gluonic heat bath. A comparison of our 1-loop perturbative calculation with lattice data suggests that the perturbative expansion is applicable for temperatures $T \gtrsim 6 T_{c}$ and distances $0.3 \lesssim R T \lesssim 1$.

\section{A. THE NON-STATIC CONTRIBU- TIONS}

We have calculated the color averaged heavy quark potential at finite temperature based on $S\left[A_{i}, A_{0}\right]=\int d x_{0} \int d^{3} x\left(-1 / 2\left(F_{0 i}^{a}\right)^{2}-1 / 4\left(F_{i j}^{a}\right)^{2}\right)$. In this expression $F_{\mu \nu}^{a}$ is the usual non-abelian field-strength tensor where $A_{i}(i=1,2,3)$ denotes the static and $A_{0}$ the non-static gauge field and we introduce a temporal gauge $\left(\partial_{0} A_{0}=0\right)$. The $g^{6}$-correction (1-loop) to the color averaged potential can be divided in a static $\left(\Delta V^{s t}\right)$ and a non-static $\left(\Delta V^{n s}\right)$ part:

$$
\Delta V_{a v}(R, T)=\Delta V^{s t}(R, T)+\Delta V^{n s}(R, T)
$$

The static contribution is well known from [5]. For the non-static part we find at 1-loop level:

$$
\begin{aligned}
& \Delta V^{n s}(R, T)= \\
& -\frac{g^{4} \beta^{2}\left(N^{2}-1\right)}{N^{2}}\left(\frac{e^{-m_{D} R}}{4 \pi R}\right) \\
& \times \int \frac{d^{3} p}{(2 \pi)^{3}}\left(\Pi_{00}^{n s}(p)-m_{D}^{2}\right) \frac{1}{\left(p^{2}+m_{D}^{2}\right)^{2}} e^{i p R},
\end{aligned}
$$

where $\Pi_{00}^{n s}(p)$ is the self-energy of the non-static field with $\Pi_{00}^{n s}(p=0)=m_{D}^{2}$ and $\beta=1 / T$. Note that in our conventions the leading order term comes from the non-static contribution. In finite temperature field theory $\Pi_{00}^{n s}(p)$ consists of the UV-divergent zero temperature part and the convergent thermal part: $\Pi_{00}^{n s}(p)=\Pi_{00}^{(T=0)}(p)+$ $\Pi_{00}^{(T)}(p) . \Pi_{00}^{(T=0)}(p)$ is calculated in [9]:

$$
\begin{aligned}
& \Pi_{00}^{(T=0)}(p) \\
& =-\frac{g^{2} N}{16 \pi^{2}}\left(\frac{11}{3} \ln \frac{4 \pi \nu^{2} e^{-\gamma_{E}}}{p^{2}}+\frac{31}{9}\right) p^{2} .
\end{aligned}
$$

The thermal contribution can be extracted from a direct integration of the loop diagrams contributing to $\Pi_{00}^{n s}(p)$ with standard calculation techniques at finite temperature. Our 1-loop calculation of $\Pi_{00}^{n s}(p)$ is in accordance with (6) and the resulting potentials are plotted in Fig. 1.

\section{REFERENCES}

1. S. Digal et al, Phys. Lett. B541 (2001) 57

2. O. Kaczmarek et al, Phys. Rev. D62 (2000) 034021

3. P. Petreczky et al, hep-lat/0103034 (2001)

4. L. G. McLarren, B. Svetitsky, Phys. Rev. D24 (1981) 450

5. S. Nadkarni, Phys. Rev. D33 (1986) 3738

6. L. S. Brown, W. I. Weisberger, Phys. Rev. D20 (1979) 3239

7. F. Zantow et al, hep-lat/0110106

8. G. Bali, K. Schilling, Phys. Rev. D47 (1993) 601

9. G. Curci, P. Menotti, Z. Phys. C21 (1984) 281 